\title{
Random vibration studies of an SDOF system with shape memory restoring force
}

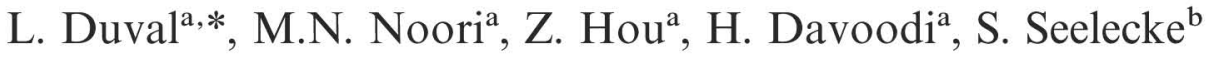 \\ ${ }^{a}$ Mechanical Engineering Department, Worcester Polytechnic Institute, Worcester, MA 01609-2280, USA \\ ${ }^{\mathrm{b}}$ Technische Universität Berlin, Institut für Verfahrenstechnik, 10623 Berlin, Germany
}

\begin{abstract}
Intelligent and adaptive material systems and structures have become very important in engineering applications. The basic characteristic of these systems is the ability to adapt to the environmental conditions. A new class of materials with promising applications in structural and mechanical systems is shape memory alloy (SMA). The mechanical behavior of shape memory alloys in particular shows a strong dependence on temperature. This property provides opportunities for the utilization of SMAs in actuators or energy dissipation devices. However, the behavior of systems containing shape memory components under random excitation has not yet been addressed in the literature. Such a study is important to verify the feasibility of using SMAs in structural systems. In this work a nondeterministic study of the dynamic behavior of a single-degree-of-freedom (SDOF) mechanical system, having a Nitinol spring as a restoring force element is presented. The SMA spring is characterized using a one-dimensional phenomenological constitutive model based on the classical Devonshire theory. Response statistics for zero mean random vibration of the SDOF under a wide range of temperature is obtained. Furthermore, nonzero mean analysis of these systems is carried out. (C) 2000 Elsevier Science B.V. All rights reserved.
\end{abstract}

Keywords: Constitutive modeling; Shape memory alloys; Random vibration; Vibration control

\section{Introduction}

Contrary to other metallic materials, which have a well-defined yield limit, SMAs can undergo large amount of inelastic deformations, without permanent plastic deformation, and recover that upon temperature change and/or loading. The fact behind this extraordinary ability, known as superelasticity, is the phase transformation mechanism. A temperature gradient and/or an externally applied load can provide the required energy for the phase transformation. These two types of transformation are referred to as temperature- or stress-induced phase transformations, respectively. In most cases for practical applications either temperature gradient or externally applied load can be treated as driving force.
The behavior of shape memory alloys and other 'smart' materials is governed by a diffusionless phase transformation between a high-temperature, low-strain austenite phase and a low-temperature, high-strain martensite phase [1]. The martensite phase is stable in lowtemperature range while the austenite phase is stable in high-temperature range. The characteristic transition temperatures, which are material properties, are $M_{\mathrm{f}}, M_{\mathrm{s}}$, $A_{\mathrm{s}}, A_{\mathrm{f}}$ : martensite finish, martensite start, austenite start and, austenite finish, respectively. These transition temperatures depend on the alloy's composition and its thermomechanical processing. Experimental evidence [2] also shows that, temperatures at which forward and reverse transformations take place depend on the state of stress and increase with stress. Austenite start and finish temperatures are more sensitive than martensite start and finish temperatures. An in depth analysis of phase transformation mechanisms and pseudoelasticity can be found in the literature [3]. 
The phenomena associated with martensitic transformations are intrinsically nonlinear. As a consequence, when subjected to dynamic inputs, a mechanical system that contains a memory element may experience a number of quite complex behaviors.

The dynamics of mechanical systems with components that exhibit a shape-memory effect is an interesting and important topic. The design of such mechanical systems is certainly made more tractable by the availability of accurate mathematical models. The purpose of this paper is to describe some efforts with a numerical simulation for one such model, and to examine some of the effects of system parameters on the random response of a mechanical system with a shape memory element. First, we briefly review the phenomenological theory that describes thermoelastic martensitic transformations in metallic alloys. This theoretical background is used to build a model of a helical spring with shape memory. The random response of an SDOF mechanical system is then investigated.

\section{Polynomial constitutive model}

Several constitutive models for SMA are available in the literature [3]. As a preliminary study, the polynomial constitutive model is used in this paper. The model is based on the Devonshire theory proposed in 1982 [4]. This one-dimensional model does not consider dissipation and represents the shape memory and pseudoelasticity effects considering a polynomial free energy which only depends on the temperature and one-dimensional strain, $\varepsilon$, i.e. $\Psi=\Psi(T, \varepsilon)$. Also, is assumed that no internal variables are considered.

The form of the free energy is chosen in such a way that the minimum and maximum points represent stability and instability of each phase of the SMA. As is usual on the one-dimensional models proposed for SMAs, $[5,6]$ three phases are considered: an austenite and two martensites $\left(M_{+}, M_{-}\right)$. Hence, the free energy is chosen such that for high temperatures it has only one minimum at vanishing strain, representing the equilibrium of the austenite phase. At low-temperatures, martensitic is stable, and the free energy must have two minimums at nonvanishing strains. At intermediate temperatures, the free energy must have equilibrium points corresponding to both phases. These restrictions are satisfied by the following polynomial expression:

$\rho \Psi(\varepsilon, T)=\frac{1}{2}\left[a_{0}+a_{1}\left(T-T_{\mathrm{M}}\right)\right] \varepsilon^{2}-\frac{1}{4} a_{2} \varepsilon^{4}+\frac{1}{6} a_{3} \varepsilon^{6}$,

where $a_{0}, a_{1}, a_{2}, a_{3}$ are positive constants, while $T_{\mathrm{M}}$ is the temperature below which the martensitic phase is stable and $\rho$ is the mass density. If we define $T_{\mathrm{A}}$ as the temperature above which the austenite is stable, and the free energy has only one minimum at zero strains, it is possible to write the following condition:

$T_{\mathrm{A}}=T_{\mathrm{M}}+\frac{1}{4} \frac{a_{2}^{2}}{a_{1} a_{3}}-\frac{a_{0}}{a_{1}}$.

Hence, the constant $a_{3}$ may be expressed in terms of the other constants of the material.

By definition [7], we have

$\sigma=\rho \frac{\partial \Psi}{\partial \varepsilon}$.

Therefore, the following stress-strain relation is obtained:

$\sigma=\left[a_{0}+a_{1}\left(T-T_{\mathrm{M}}\right)\right] \varepsilon-a_{2} \varepsilon^{3}+a_{3} \varepsilon^{5}$.

When an external stress field $\bar{\sigma}$ is applied, it is convenient to consider the total free energy, $\Psi$ as follows:

$\rho \Psi=\rho \psi-\bar{\sigma} \varepsilon$.

It should be emphasized that this model considers one free energy, with no extra internal variables, to represent phase transformations on SMAs with an austenitic phase and two variants of martensitic. This characteristic makes the polynomial constitutive model a simple alternative to describe SMAs behavior. The absence of experimental tests to determine the material constants is one of the greatest difficulties in the use of this model.

The original Devonshire model can be obtained by just taking $a_{0}=0$ in the polynomial expression given by Eq. (4), and recalling that without dissipation the stress is simply the derivative of the free energy with respect to strain, we obtain the constitutive law based on the Devonshire theory:

$\sigma=a_{1}\left(T-T_{\mathrm{M}}\right) \varepsilon-a_{2} \varepsilon^{3}+\frac{1}{4} \frac{a_{2}^{2}}{a_{1}\left(T_{\mathrm{A}}-T_{\mathrm{M}}\right)} \varepsilon^{5}$.

The Devonshire theory can describe shape memory and pseudoelasticity qualitatively well. However, the slope of the stress-strain curve at the origin varies linearly with temperature. This means that on small strain approximation, the Devonshire theory would predict an elastic modulus that will vary with temperature.

\section{A simple system with shape memory}

The constitutive model discussed in the preceding section can be used to model helical springs made of shape memory alloys. In order to model the response of an SDOF system which includes a helical spring made from shape memory alloy, we can apply the constitutive model discussed in the preceding section. In fact, this theory provides a fairly good qualitative description of the onedimensional shape memory and pseudoelastic effects. The Devonshire's theory yields the simplest equation for the nonlinear restoring force. 
Let us assume that the longitudinal external force is resisted by the torsional shear stresses developed on the circular cross section of a helical shape wire $[7,8]$. The relationship between the longitudinal force, $F$, and the shear stress distribution, $\sigma$, is expressed as

$F=\frac{4 \pi}{D} \int_{0}^{d / 2} \sigma r^{2} \mathrm{~d} r$

where $r$ is the radial coordinate along the cross section, while $D$ and $d$ represent, respectively, the mean diameter and the wire diameter of the spring. We also assume that the shear strain $\gamma$ is distributed linearly along the wire cross section. If $N$ is the number of coils and $X$ the displacement, it can be shown [7] that

$\gamma=\frac{2 r}{\pi D^{2} N} X$

Now, using the polynomial model, and assuming that Eq. (6) is valid for the pure shear stress-strain behavior, we obtain the following nondimensional equation for the nonlinear restoring force

$f=(\theta-1) x-\lambda x^{3}+\frac{\lambda^{2}}{4\left(\theta_{\mathrm{c}}-1\right)} x^{5}$,

where $f$ and $x$ are the nondimensional force and the displacement and are given as

$f=\frac{8 D}{\pi a_{1} T_{\mathrm{M}} d^{3}} F$,

$x=\frac{d}{\pi D^{2} N} X$.

The other parameters are defined as

$\theta=\frac{T}{T_{\mathrm{M}}}$,

$\lambda=\frac{2 a_{2}}{3 a_{1} T_{\mathrm{M}}}$,

$\theta_{\mathrm{c}}=\frac{1}{9}\left(8 \frac{T_{\mathrm{A}}}{T_{\mathrm{M}}}+1\right)$

In order to study the behavior system with shape memory component, an SDOF system is considered. The system consists of a mass $m$, a shape memory spring and a linear damping $c$, the excitation force is $P \sin (\omega t)$. The equation of motion in a nondimensional form can be written as

$\ddot{x}+\zeta \dot{x}+(\theta-1) x-\lambda x^{3}+\frac{\lambda^{2}}{4\left(\theta_{c}-1\right)} x^{5}=\alpha \sin (\Omega \tau)$.
The dots represent derivatives with respect to the nondimensional time $\tau$, and the constants are defined as

$\tau=\omega_{0} t$,

$\omega_{0}=\left[\frac{a_{1} T_{\mathrm{M}} d^{4}}{8 m D^{3} N}\right]^{1 / 2}$,

$\zeta=\frac{c}{m \omega_{0}}$,

$\alpha=\frac{8 D}{\pi a_{1} T_{\mathrm{M}} d^{3}} P$,

$\Omega=\frac{\omega}{\omega_{0}}$.

Using a sate-space form and defining $y_{1}=x$ and $y_{2}=\dot{x}$, Eq. (12) can be written as a set of first-order differential equations:

$$
\begin{aligned}
\dot{y}_{1}= & y_{2}, \\
\dot{y}_{2}= & -(\theta-1) y_{1}+\lambda y_{1}^{3}-\frac{\lambda^{2}}{4\left(\theta_{\mathrm{c}}-1\right)} y_{1}^{5}-\zeta y_{2} \\
& +\alpha \sin (\Omega \tau) .
\end{aligned}
$$

A standard fourth-order Runge-Kutta was used to numerically integrate Eqs. (14a) and (14b). We have obtained good convergence with a step size $\Delta t=2 \pi / 180$.

\section{Numerical results}

In this section the response of the shape memory oscillator, subjected to random excitation, is discussed. The system is governed by Eqs. (14a) and (14b), where $\alpha$ and $\Omega$ are the nondimensional parameters representing, respectively, the amplitude and the frequency of the forcing function. Since we are more concerned with the qualitative response of the shape memory system, $\lambda=1$ has been chosen for the numerical studies presented throughout this paper. We also assume $\theta_{\mathrm{c}}=1.1$ which is a typical value for NiTi alloy [9].

Due to space limitation only the displacement responses are presented. Nonzero and zero mean analyses have been performed. In both cases, the behavior of the system when subjected to a temperature variation has been obtained by assuming that the temperature of the system initially $\theta_{\mathrm{i}}$ changes to $\theta_{\mathrm{f}}$ in the following way [7]:

$\theta= \begin{cases}\theta_{\mathrm{i}} & t \leqslant t_{\mathrm{i}}, \\ \theta_{\mathrm{i}}+\left(\theta_{\mathrm{f}}-\theta_{\mathrm{i}}\right) \sin \left[\frac{\pi}{2}\left(\frac{t-t_{\mathrm{f}}}{t_{\mathrm{f}}-t_{\mathrm{i}}}\right)\right], & t_{\mathrm{i}}<t<t_{\mathrm{f}}, \\ \theta_{\mathrm{f}} & t \geqslant t_{\mathrm{f}} .\end{cases}$

Temperature variation is induced when the mass of the system is displaced from its equilibrium position at instant $t=0$ and after an interval of time $t_{\mathrm{i}}(10 \mathrm{~s})$, the 


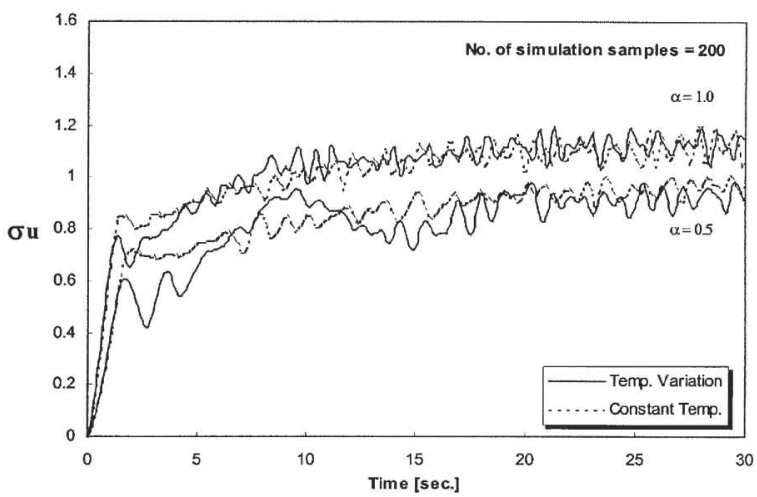

Fig. 1. Nonzero mean RMS displacement response of an SDOF nondissipative system $(\zeta=0)$ under stationary white noise input $S_{0}=1.0$ and $\mu_{\mathrm{f}}=0.8$.

temperature begins to change, up to the instant $t_{\mathrm{f}}(20 \mathrm{~s})$, when it reaches the final temperature. The chosen temperature variation law obeys a simple assumption that this varies in a sinusoidal fashion in the pre-defined time interval. Dissipative and non-dissipative system conditions have also been considered in the analysis. Fig. 1 shows the nonzero mean analysis for nondissipative system $(\zeta=0)$ with $S_{0}=1, \mu_{\mathrm{f}}=0.8$ (mean excitation value) and different temperature conditions. Initially, we observe the statistical response for two different levels of forcing amplitudes $(\alpha=1.0$ and 0.5$)$. As the forcing parameter $\alpha$ increases, the system dynamics becomes richer and the effect of temperature variation reduces the displacement system response. The time history response of the dissipative system $(\zeta=0.2)$ for zero mean excitation $\left(\mu_{\mathrm{f}}=0\right)$ analysis is shown in Fig. 2. As expected the displacement response is reduced due to both damping effect and temperature variation. This behavior is of special interest, since it illustrates the capability of altering the dynamics of the shape memory system by changing its temperature. This feature has motivated the use of shape memory actuators for active vibration control.

\section{Conclusions}

In this paper we have focused on the qualitative statistical response of a simple dynamical system with shape

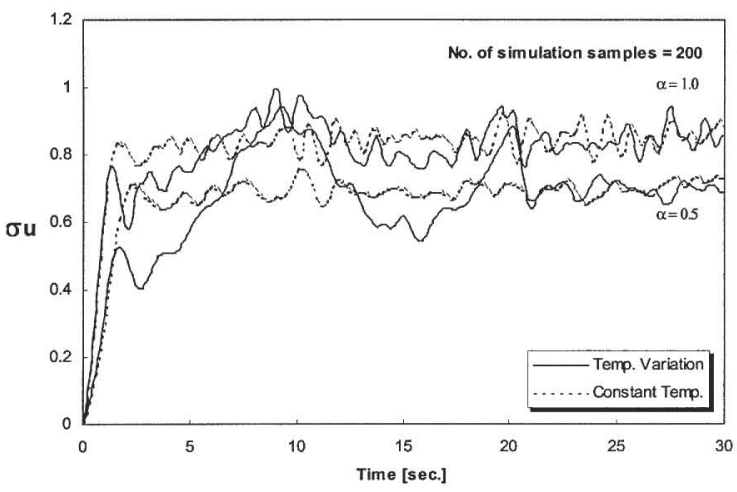

Fig. 2. Zero mean RMS displacement of an SDOF dissipative system $(\zeta=0.2)$ under stationary white noise input $S_{0}=1.0$ and $\mu_{\mathrm{f}}=0$.

memory. The constitutive theory that assumes a polynomial expression for the free-energy functional has been used to model a helical spring with shape memory. This choice yields the simplest analytical form for the nonlinear restoring force. The statistical response of an SDOF system containing such a spring has been studied numerically.

\section{References}

[1] O. Bruno, in: H.T. Banks (Ed.), SPIE 2192 (1994) 370.

[2] L. Delaey et al., Mat. Sc. 9 (1974) 1521.

[3] L. Duval, et al., in: N. Hagood, M. Atalla (Eds.), Ninth International Conference on Adaptive Structures and Technologies, Technomic, Lancaster, 1998, pp. 260.

[4] F. Falk, J. Phys. C4 12-43 (1982) 3.

[5] K.H. Hoffman et al., Report No. 201, Universität Augsburg, Germany, 1990.

[6] H.W. Alt et al., Report No. 90, Universität Augsburg, Germany, 1990.

[7] M.A. Savi et al., Int. J. Modeling Simulation 1998, in submission.

[8] E.J. Shigley, Mechanical Engineering Design, McGrawHill, New York, 1972.

[9] H. Tobushi et al., JSME Int. J. 34 (1990) 83. 